\title{
Competencia para el cuidado y acceso a la salud rural
}

\author{
Competence for care and access to rural health \\ Competência para o atendimento e acesso à saúde rural
}

\author{
Lorena Alejandra Bernal-Barón ${ }^{1}$ \\ Olga Janneth Gómez-Ramirez ${ }^{2}$
}

\section{Resumen}

Objetivos: Describir la competencia para el cuidado de la persona con enfermedad crónica y su cuidador, residentes en zona rural e identificar las barreras que limitan el acceso a los servicios de salud. Materiales y Métodos: estudio cuantitativo de corte transversal, con una muestra no probabilística de 218 diadas (paciente -cuidador), que cumplieron con los criterios de inclusión del estudio y a la que se aplicaron los siguientes instrumentos: Ficha de caracterización de la diada; Competencia para el cuidado en el hogar paciente y cuidador y Encuesta de acceso a servicios de salud para hogares colombianos. Resultados: La competencia para el cuidado del paciente revela ser menor que la desarrollada por el cuidador. Sin embargo, en ambos casos la mayor deficiencia en los residentes rurales es la falta de conocimientos sobre la patología crónica que se padece, de esta manera se convierte en un reto para el cuidado en la ruralidad. De igual manera, se hace evidente que el acceso a los servicios de salud es limitado en estas poblaciones, dado por barreras de acceso de tipo administrativo, económico y de desplazamiento que se extienden por las condiciones propias de la zona rural. Ante dicho escenario, la enfermera (o) se transforma en el profesional idóneo y con las capacidades adecuadas para mitigar desde su actuar estas dificultades, mediante el reconocimiento de las condiciones iniciales de la población y la gestión de estrategias que permitan que los programas de salud de la instituciones puedan llegar a las poblaciones más vulnerables. Conclusión: En la ruralidad, los retos son diversos y adversos, sin embargo, se hace necesaria su intervención, con el objetivo de mejorar las condiciones de salud en las poblaciones que allí residen.

Palabras clave: Enfermedad Crónica; Accesibilidad a los Servicios de Salud; Población Rural; Cuidadores.
Autor de correspondencia*

${ }^{1 *}$ Enfermera, Magister en enfermería. Tunja, Colombia Correo: lbernalb@unal.edu. co (iD)

${ }^{2}$ Enfermera, Doctora en Enfermería. Profesora Asociada. Facultad de Enfermería. Universidad Nacional de Colombia. Bogotá, Colombia. Correo: ojgomezr@unal. edu.co (iD)

\section{Abstract}

Objectives: To describe the competence for the care of the chronically ill person and their caregiver, rural residents, and to identify the barriers that limit access to health services. Materials and Methods: Quantitative cross-sectional study, with a non-pro-

(c) Universidad Francisco de Paula Santander. Este es un artículo bajo la licencia CC-BY-NC-ND (ㄷ)(1) (3)

Recibido: 20 marzo 2020 Aprobado: 7 julio 2020

Para citar este artículo/ To reference this article/ Para citar este artigo/

Bernal-Barón LA, Gómez-Ramírez OJ. Competencia para el cuidado y acceso a la salud rural. Rev. cienc. cuidad. 2020; 17(3):46-60. https://doi.org/10.22463/17949831.2210 
babilistic sample of 218 dyads (patient-caregiver), which met the study's inclusion criteria and to which the following instruments were applied: Dyad characterization sheet; Competence for care in the patient and caregiver home and Survey of access to health services for Colombian households. Results: The competence for the care of the patient revealed to be less than that developed by the caregiver. However, in both cases the greatest deficiency in rural residents is the lack of knowledge about the chronic pathology they suffer, thus making it a challenge for care in rural areas. Similarly, it is evident that access to health services is limited in these populations, given the administrative, economic and displacement barriers to access that are spread by the conditions of the rural area. Faced with this scenario, the nurse becomes the ideal professional with the adequate capacities to mitigate these difficulties through the recognition of the initial conditions of the population and the management of strategies that allow the health programs of the institutions to reach the most vulnerable populations. Conclusion: In rural areas, the challenges are diverse and adverse; however, it is necessary to intervene in order to improve the health conditions of the populations living there.

Keywords: Chronic disease; Accessibility to health services; rural population; caregivers.

\begin{abstract}
Resumo
Objetivos: Descrever a competência para o cuidado do doente crônico e de seu cuidador, os residentes rurais e identificar as barreiras que limitam o acesso aos serviços de saúde. Materiais e Métodos: Um estudo quantitativo transversal, com uma amostra não-probabilística de 218 díades (paciente cuidador), que atendeu aos critérios de inclusão do estudo e ao qual foram aplicados os seguintes instrumentos: Cartão de caracterização dos díades; Competência para o atendimento no domicílio do paciente e cuidador e Pesquisa de Acesso aos Serviços de Saúde para domicílios colombianos. Resultados: A competência para o atendimento ao paciente revelou-se inferior à desenvolvida pelo cuidador. Entretanto, em ambos os casos, a maior deficiência dos residentes rurais é a falta de conhecimento sobre a patologia crônica que eles sofrem, tornando-se assim um desafio para o cuidado nas áreas rurais. Da mesma forma, é evidente que o acesso aos serviços de saúde é limitado nessas populações, dadas as barreiras administrativas, econômicas e de deslocamento ao acesso que são disseminadas pelas condições da área rural. Diante deste cenário, a enfermeira torna-se o profissional ideal com as habilidades apropriadas para mitigar estas dificuldades, reconhecendo as condições iniciais da população e gerenciando estratégias que permitem que os programas de saúde das instituições cheguem às populações mais vulneráveis. Conclusão: Nas áreas rurais, os desafios são diversos e adversos; entretanto, sua intervenção é necessária, com o objetivo de melhorar as condições de saúde das populações que nelas residem.
\end{abstract}

Palavras-chave: Doença crônica; Acessibilidade aos serviços de saúde; população rural; cuidadores

\section{Introducción}

La zona geográfica en donde residen las personas influye en diversos aspectos de la vida en los que no son ajenos a esta situación la capacidad de cuidado y el acceso a la salud. Este es el caso de la zona rural cuya población ha sido considerada en diferentes escenarios, como una población en condición de vulnerabilidad. En la zona rural las condi- ciones a las cuales se enfrentan las personas son adversas. Sumado a lo anterior, los factores asociados a la gestión de la enfermedad convergen y se hacen más complejos, lo que genera que la carga de la enfermedad sea mayor cuando la persona reside en una región rural (1).

Lo anterior repercute en la condición de salud de la población conllevando a que el cuidado de la enfermedad por parte del paciente sea más difícil. Desafortunadamente, una población rural típica presenta factores como el bajo 
nivel educativo y baja situación socioeconómica. Asociado a estos factores también pueden presentar antecedentes de enfermedad crónica (EC) y edad avanzada; los cuales, son clasificados como factores de riesgo para el empeoramiento de la EC (2,3). En general, la cronicidad de una patología repercute y a la vez se ve influenciada por diferentes aspectos del entorno.

Es conocido que la patología crónica, no solo impacta la vida de la persona que la padece, sino también en la dinámica familiar del enfermo. Así, la EC se define como un proceso de evolución prolongada, que no se resuelve espontáneamente y rara vez alcanza una cura completa. La EC genera una gran carga social tanto desde el punto de vista económico como desde la perspectiva de dependencia social e incapacidad (4). Es así como la persona con EC que vive en zona rural, no se cuida sólo en las instituciones de salud, sino también en casa al lado de un cuidador. En el hogar, el paciente crónico y su cuidador se encuentran en un escenario donde la falta de conocimientos y las barreras en el acceso a los servicios de salud pueden afectar su capacidad de cuidado.

Para realizar la medición de dicha capacidad, tanto en la persona con EC como en su cuidador, se utilizó la competencia para el cuidado en el hogar, que se define como la capacidad, habilidad y preparación que tiene la persona con EC y/o el cuidador familiar para ejercer la labor de cuidar en el hogar (5). Esta labor se genera desde una perspectiva integral en la que se identifican aspectos de conocimiento de la enfermedad, condiciones personales, habilidades instrumentales, capacidad de anticiparse, factores básicos de bienestar y disfrutar, interacción social y redes de apoyo (6).

Este fenómeno ha sido ampliamente estudiado en el territorio nacional, con diferentes poblaciones $\mathrm{y}$ variadas regiones, en donde cabe resaltar el estudio de Carrillo et al. (5) realizado en las diferentes regiones de Colombia, ya que es el único estudio en el país, en donde se hace distinción de la población rural. Sin embargo, es evidente la carencia de estudios que profundizan este fenómeno en la ruralidad

Así las cosas, se evidencia que el cuidado de la salud y la enfermedad no dependen únicamente del enfermo crónico y/o su cuidador, sino que además el sistema de salud juega un papel indispensable desde el punto de vista del acceso. $\mathrm{Si}$ el enfermo crónico cuenta con una óptima accesibilidad a los servicios de salud, este, tendrá la oportunidad de adquirir conocimientos, acceso a la atención médica y de otros profesio- nales, oportunidad frente a la recepción de su tratamiento farmacológico, entre otras actividades necesarias para su cuidado.

Sin embargo, esto se convierte en muchas ocasiones en una utopía, puesto que lo vivido es un reto constante para lograr el acceso a la salud. Es importante anotar, que el sistema de salud vigente no fue diseñado teniendo en cuenta las particularidades de las poblaciones rurales y los riesgos a los que se enfrentan (7). El panorama es menos alentador para las poblaciones más vulnerables, como lo son las poblaciones rurales, pobres e indígenas, ya que son las que tienen peor acceso a los sistemas de salud (8). Los problemas de accesibilidad y atención tienden a ser peor en las zonas rurales que en las zonas urbanas debido al desarrollo económico desequilibrado y una distribución desigual de los recursos sanitarios (9).

Según las bases del Plan Nacional de Desarrollo, aún persisten barreras e inequidades de acceso real y efectivo de los usuarios a los servicios de salud. Estos obstáculos se deben entre otros, a aspectos geográficos entre lo rural y lo urbano, así como habitar en zonas de alta dispersión poblacional (10). El acceso al servicio de salud en la zona rural es un problema en zonas como América latina. Este problema ha sido previamente demostrado como un factor importante relacionado con la calidad de la evaluación de individuos con trastornos crónicos que viven en zonas rurales y remotas (3).Las disparidades de salud adicionales que experimentan las personas que viven en zonas rurales tales como la desigualdad económica, envejecimiento de la población, los problemas de transporte y servicios de salud limitados, pueden tener un impacto mayor sobre cómo las personas son capaces de manejar sus condiciones crónicas (1).

A nivel de Colombia y según el informe nacional de calidad de la atención en salud, para el 2014, la percepción de la facilidad de acceso a los servicios de salud fue de 54\%, lo que indica que el $46 \%$ de los usuarios consideraron difícil acceder a los servicios de salud (11). Por otra parte, según el Boletín Técnico de Pobreza Multidimensional en Colombia año 2018, el porcentaje de hogares con barreras de acceso a los servicios de salud fue de $6,2 \%$ (12). Esto refleja la inequidad en salud existente en el país y la necesidad sentida de intervenir en este tópico; frente a lo cual, se han generado investigaciones que coinciden en que las barreras de acceso a los servicios de salud en Colombia están asociadas a cobertura limitada del seguro de salud, ingresos o educación deficientes y características propias de los servicios 
tales como accesibilidad geográfica, asuntos administrativos o calidad (13).

En contraposición, desde la perspectiva del usuario con una EC la atención es fraccionada, desintegrada y dificulta los procesos, exigiendo múltiples autorizaciones para diferentes problemas relacionados con la EC. No se considera ni apoya a los cuidadores familiares, quien no se encuentra preparado para abordar la EC, incrementa las transiciones innecesarias y con ello el costo, el riesgo y la inseguridad de las personas con EC. Igualmente, no se tienen propuestas para ajustarse a la realidad que vive el país frente al incremento de estas enfermedades y (14) es notoria la falta de estudios al respecto en el entorno rural (7).

De esta manera, es evidente que el acceso a los servicios de salud es un factor fundamental para que la persona con EC y su cuidador puedan adquirir los conocimientos, habilidades e incluso el apoyo necesario para ser competentes frente al cuidado de la enfermedad. Es preciso en primera instancia identificar los problemas con el fin de poder intervenirlos y modificarlos. Es así como la enfermera con un adecuado nivel profesional y académico, posee las capacidades para la realización de cambios en el estado de salud de las poblaciones por medio de la identificación de limitantes frente al acceso y la deficiencia de herramientas para afrontar el cuidado de la EC. Lo que conlleva a cambios en la forma de prestar la atención en salud e incluso personalizarla, prestando especial atención a aquellas poblaciones en condición de vulnerabilidad, dentro de las cuales se incluyen las poblaciones rurales, puesto que estas regiones disponen no solo de un número restringido de médicos y enfermeras, sino también de una limitada capacidad y autonomía para prestar los servicios de atención primaria de salud necesarios (15).

\section{Objetivos}

- Describir la competencia para el cuidado de la persona con enfermedad crónica y su cuidador, residentes en zona rural.

- Identificar las barreras presentes para el acceso a los servicios de salud en la zona rural.

\section{Materiales y Métodos}

Estudio cuantitativo, de corte transversal, realizado en el programa de atención al paciente crónico de un hospital de primer nivel del municipio de Villa de Leyva, Boyacá (Colombia).
La población diana del estudio fueron las diadas constituida por persona con EC - cuidador familiar, residentes en área rural. Se utilizó como marco muestral, la base de datos de la institución, la cual contó con un total de 500 pacientes atendidos por la institución al momento de realizar el estudio. A partir de dicho marco muestral, se realizó el cálculo de la muestra con un porcentaje de confiabilidad del $95 \%$, por lo cual se obtuvo un tamaño muestral de 218 diadas. El tipo de muestreo realizado fue no probabilístico, ya que la aplicación de los instrumentos se realizó en entrevista presencial con los participantes de acuerdo al orden de llagada a las sesiones programadas por la institución durante el primer semestre de 2019 y quienes cumplieron con los criterios de inclusión.

En el estudio se incluyeron pacientes mayores de 18 años, con diagnostico medico de EC no transmisible, donde se incluyeron los diagnósticos de diabetes mellitus, hipertensión arterial y enfermedad renal crónica, sin deterioro neurológico ni cognitivo. Se excluyeron los pacientes que, a pesar de cumplir con los criterios de inclusión, cursaran un proceso agudo de su patología de base, sin cuidador familiar o residente en hogar geriátrico. En la elección de cuidador familiar, se tuvo en cuenta que este fuera mayor de 18 años y con un tiempo de más de 3 meses en el rol del cuidador.

Inicialmente se realizó una prueba piloto, en la cual se encontró que los instrumentos a utilizar eran claros para los participantes y que además permitían recolectar la información necesaria para el estudio. Dichos instrumentos son la ficha de Caracterización de la Diada cuidador-persona con enfermedad crónica del grupo de cuidado paciente crónico de la Universidad Nacional de Colombia, la cual permitió recolectar los datos de identificación sociodemográfica y clínica tanto del paciente como del cuidador. El siguiente, fue el instrumento Competencia para el cuidado en el hogar de la persona con enfermedad crónica / Cuidador familiar en su versión corta, mediante el cual se realizó la medición de la competencia individual de cada componente de la diada. Dicho instrumento cuenta con un Índice de Validez de Contenido de 0.97 y un alfa de Cronbach de 0,928. Finalmente, la Encuesta de acceso a servicios de salud para hogares colombianos (EASSS) la cual cuenta con Validación de contenido e Índice de acuerdo $>90 \%$, mediante esta encuesta, se midieron las posibles barreras de acceso a los servicios de salud que pudieran ser identificados por la diada. 
La captación de los participantes se realizó durante las jornadas de atención a pacientes con EC, programadas por la institución de salud, durante las cuales el investigador principal, realizo abordaje de los participantes por medio de entrevista directa en donde se realizó el debido diligenciamiento de los instrumentos.

Para el procesamiento de los datos, se empleó una base de datos inicial en donde se recopilaron los datos y donde además se realizó un riguroso control de calidad con el fin de reducir los posibles sesgos de información; luego, se utilizó el software IBM SPSS, en donde se procesaron los datos y se calcularon medidas de tendencia central, dispersión y frecuencias, para el análisis descriptivo. competencia para cuidar en el hogar se analizó a partir de los niveles de estratificación alto, medio y bajo.

Se tuvo en consideración los aspectos éticos contemplados en la Resolución 008430 de 1993, los principios éticos de la Ley 911 de 2004 y las pautas del Comité de Organizaciones de las Ciencias Médicas (CIOMS). El estudio fue aprobado por el comité de ética de la Universidad Nacional de Colombia y de la institución de salud donde se realizó el estudio, adicional a lo anterior, se contó con la autorización de los respectivos investigadores para el uso de los diferentes instrumentos empleados en la recolección de información. De igual manera, se contó con el consentimiento informado de los participantes en donde se expuso el riesgo mínimo que les representaba el estudio.

\section{Resultados}

En cuanto al grupo de pacientes, se encontró que el 78,4\% eran mujeres, con edad entre los 60 a 74 años, con un mínimo de edad de 31 años y un máximo de 93 años. Las principales EC encontradas fueron: Hipertensión arterial $(64,2 \%)$, enfermedad renal crónica $(33,1 \%)$ y diabetes mellitus $(2,8)$. El tiempo con la enfermedad fue de 5 a 10 años para el $28 \%$ y de 1 a 5 años para el $24,3 \%$. Se evidenció un nivel educativo bajo ya que el $33,9 \%$ de los participantes contaba con primaria incompleta y $24,3 \%$ era analfabeta; en cuanto al estado civil, el $27,6 \%$ se encontraban casados y un $25,7 \%$ se encontraba viudo; en cuanto a ocupación, el 69,3\% se dedicaba al hogar, un 56.4\% de los sujetos pertenecían al estrato socioeono- mico 1. En cuanto al sistema de aseguramiento en salud se evidenció que el 77,5\% pertenece al régimen subsidiado y el $70,6 \%$ de los pacientes cuenta con ingresos menores a un salario mínimo legal vigente.

También se midió la red de apoyo dada por los cuidadores, evidenciando que el $66,1 \%$ de los pacientes contaba con más de un cuidador. Por otra parte, el 63,8\% no requería ayuda diaria. Como principal cuidador se identificó hijo (a) con un $62,8 \%$, sin embargo, es importante reconocer que se identificaron también otros cuidadores como lo son hermano, nieto (a), nuera, primo (a), y sobrino(a). Adicionalmente, la autopercepción de carga del paciente fue predominantemente baja en el $69,3 \%$ de los sujetos.

En cuanto al uso de TIC's (nivel de conocimiento, acceso y frecuencia de uso), se encontró que los medios de información como la televisión $(47,2 \%)$ y la radio $(28,8 \%)$ son los más utilizados. El 77,5\% de los pacientes refirieron que no se apoyaban en las TIC's para asumir su cuidado.

En relación al grupo de cuidadores se encontró que el $70,6 \%$ de los cuidadores eran mujeres, con una edad entre los 35 a 59 años, con un mínimo de edad de 18 años y un máximo de 79 años. En cuanto al grado de escolaridad, el 21,6\% era profesional y con estado civil casado (a) en el $48,6 \%$ de los cuidadores. En cuanto a ocupación, el $34,4 \%$ se dedicaba al hogar, con estrato socioeconómico 1 en el 50,0\% de los sujetos. Por otra parte, el $51,4 \%$ de los cuidadores estaba a cargo de la persona con EC desde el diagnóstico de la enfermedad y el 53,7\% llevaba como cuidador entre 1 a 5 años. En cuanto a la percepción de la carga, el 90,4\% considera que no presentan sobrecarga por el cuidado.

Respecto al uso de TIC's (nivel de conocimiento, acceso y frecuencia de uso), se encontró que, los medios de información como la televisión $(57,8 \%)$, la radio $(38,1 \%)$ y el teléfono $(37,2 \%)$ son los más utilizados. El 78,0\% de los cuidadores refirieron que no se apoyaban en las TIC's para asumir el cuidado de su familiar.

Al medir la competencia para el cuidado en la diada, se indago sobre las 6 dimensiones que la conforman tanto en la persona con EC (gráfico 1) como en el cuidador familiar (gráfico 2). 


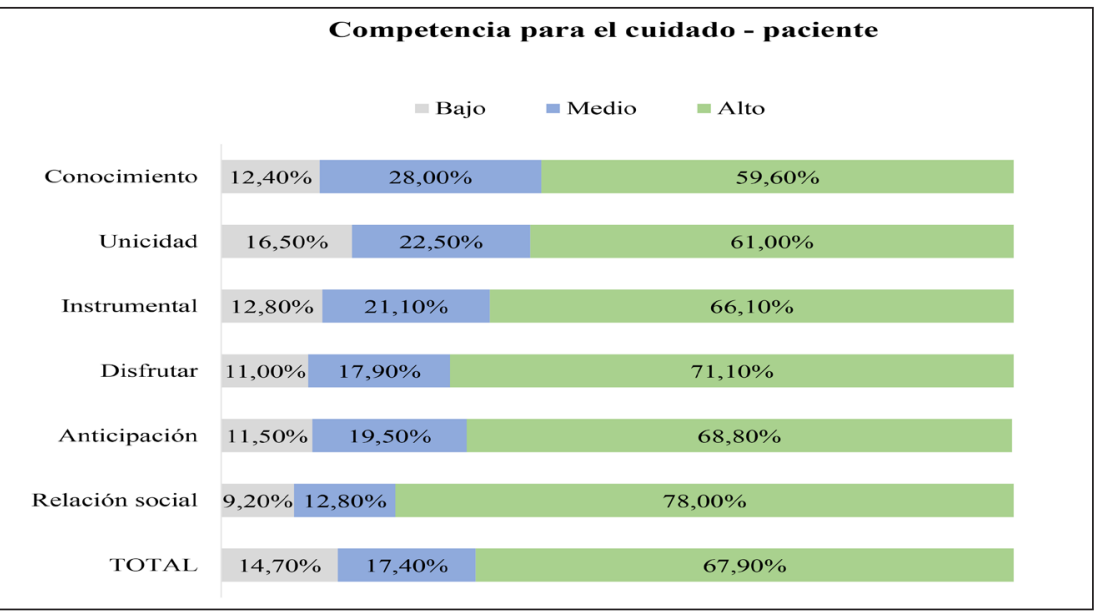

ISSN-PRINT

$1794-9831$

E-ISSN 2322-7028

Vol. 17 No. 3

Sep - Dic 2020

Cúcuta, Colombia

Gráfico 1. Competencia para el cuidado en el hogar del paciente con EC en zona rura Fuente: Elaboración propia

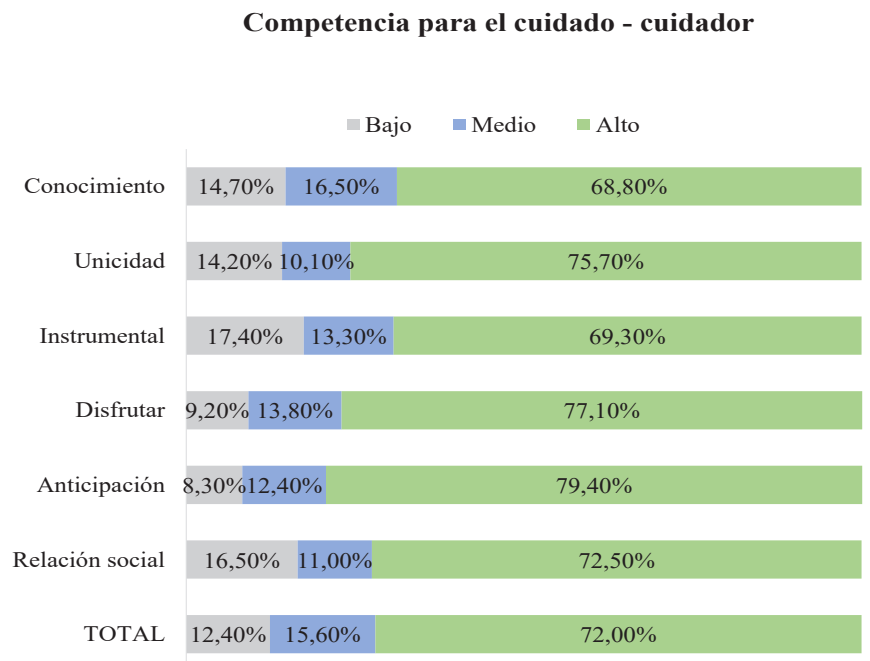

Gráfico 2. Competencia para el cuidado en el hogar del cuidador familiar del pacient con EC en zona rural

Fuente: elaboración propia

En general la competencia para el cuidado se encontró en nivel alto en el $67,9 \%$ de los pacientes con enfermedad crónica entrevistados, además, la dimensión con mejor puntuación, fue la de relación social. En el caso de los cuidadores familiares, la competencia para el cuidado se encontró en nivel alto en el $72,0 \%$ de los cuidadores entrevistados y para este grupo la dimensión con mejor puntuación fue anticipación. Adicional a lo anterior, se resalta que la dimensión conocimiento fue la mas baja en ambos grupos.

En cuanto al acceso que presentan los pacien- tes con EC que residen en zona rural, se realizó la medición del acceso a servicios preventivos, encontrando, que más del $90 \%$ de los pacientes acuden a servicios tales como consulta de quinquenio, toma de paraclínicos de prevención y toma de tensión arterial. En cuanto a desplazamiento desde su lugar de residencia a la institución de salud, el tiempo de llegada en el 86,7 \% de los casos de menos de 1 hora y el 13,3\% de 1 a 4 horas. Los medios de transporte utilizados se explican en la tabla 1. 
Tabla 1. Medio de transporte utilizado

\begin{tabular}{cc}
\hline Medio de transporte & Porcentaje \\
\hline caminata & $37,6 \%$ \\
bus & $26,1 \%$ \\
vehículo propio & $13,3 \%$ \\
caminata y bus & $11,9 \%$ \\
taxi & $9,6 \%$ \\
bicicleta & $1,4 \%$ \\
\hline
\end{tabular}

Fuente: Elaboración propia

Frente al acceso a servicios curativos o rehabilitación, el 23,4\% de los pacientes asistieron al servicio de urgencias, en cuyos casos, el tiempo de atención fue inmediato $(7,8 \%)$, de máximo 30 minutos $(66,75)$, entre 31 minutos a 1 hora $(21,6 \%)$ y de 1 a 2 horas $(3,9 \%)$. Frente a este medio de consulta, los pacientes consideraron que recibieron la atención necesaria $(84,3 \%)$ y califican la atención como muy buena $(19.6 \%)$, buena $(70,6 \%)$, y mala $(9,8 \%)$. El pago de la atención fue por medio de la EPS tipo subsidiada $86,3 \%$ y de tipo contributivo $13,7 \%$.

Frente a la consulta por médico general el 79,8\% asistió a dicha atención, con un tiempo de espera para la adquisición de la cita de el mismo día $61,5 \%$. La totalidad de los pacientes que consultaron, consideraron que le fue ofrecida la atención necesaria y el 95,5\% refiere no haber presentado problemas para la entrega del tratamiento. La fuente económica para cubrir costos de consulta por médico general fue por EPS subsidiado en el $70,7 \%$, contributivo en el $13,8 \%$ y régimen especial en el $14,9 \%$. Del total de los pacientes que acudieron a consulta médica, el 66,7\% fue remitido a algún especialista, de los cuales se resalta medicina interna en el $29,8 \%$ de los casos.

En la tabla 2, se observa el tiempo de espera referido por los pacientes, para la autorización de la EPS para la consulta por medicina especializada.

Tabla 2. Tiempos de espera para autorización de consulta por especialista

\begin{tabular}{lc}
\hline $\begin{array}{l}\text { Tiempo entre solicitud de } \\
\text { cita con la especialidad y } \\
\text { la autorización de la EPS }\end{array}$ & $\begin{array}{c}\text { Porcentaje de } \\
\text { pacientes }\end{array}$ \\
\hline el mismo día & $12,1 \%$ \\
menos de 1 semana & $55,2 \%$ \\
entre 2 a 4 semanas & $25,9 \%$ \\
entre 1 a 3 meses & $6 \%$ \\
no lo había tramitado & $0,9 \%$ \\
\hline
\end{tabular}

Fuente: Elaboración propia
En la tabla 3, se observa el tiempo de espera referido por los pacientes para la obtención de la cita efectiva, luego de la autorización de la EPS

Tabla 3. Tiempos de espera para obtención de consulta por especialista

\begin{tabular}{lc}
\hline $\begin{array}{l}\text { Tiempo entre la autori- } \\
\text { zación de la EPS y la cita } \\
\text { efectiva }\end{array}$ & $\begin{array}{c}\text { Porcentaje de } \\
\text { pacientes }\end{array}$ \\
\hline el mismo día & $0,9 \%$ \\
menos de 1 semana & $13,8 \%$ \\
entre 2 a 4 semanas & $39,7 \%$ \\
entre 1 a 3 meses & $27,6 \%$ \\
entre 3 a 6 meses & $5,2 \%$ \\
más de 6 meses & $3,4 \%$ \\
no ha sido atendido & $9,5 \%$ \\
\hline
\end{tabular}

Fuente: Elaboración propia

De los pacientes que fueron atendidos por médico especialista el 85,3\% recibió la atención necesaria, en cuanto al requerimiento de medicamentos. Al 94,3\% le fue formulada medicación y en el 7,8\% de estos casos no fueron entregados los medicamentos, debido a que el medicamento no está en cobertura por el POS, no había medicamentos o no gestiono la entrega de estos. En general los pacientes consideraron que la calidad de la atención por medicina general y especialista fue $26,6 \%$ muy buena, $62,3 \%$ buena, $9,2 \%$ mala y $1,7 \%$ no sabe no responde.

En caso de los pacientes que no acudieron a consulta por medicina general, los aspectos que influyeron fueron variados, como se muestra en la tabla 4.

Tabla 4. Motivos de inasistencia a consulta medica

\begin{tabular}{ll}
\hline \multicolumn{1}{c}{ Motivo de no consulta } & Porcentaje \\
\hline $\begin{array}{l}\text { Falta de compañía o no lo con- } \\
\text { sideró necesario }\end{array}$ & $43,9 \%$ \\
$\begin{array}{l}\text { Falta de dinero para transportes } \\
\text { y copagos }\end{array}$ & $17,1 \%$ \\
$\begin{array}{l}\text { Consultó antes y no le resolvie- } \\
\text { ron el problema }\end{array}$ & $12,2 \%$ \\
Falta de tiempo & $9,8 \%$ \\
Tramites excesivos & $7,3 \%$ \\
$\begin{array}{l}\text { El centro de atención queda } \\
\text { lejos }\end{array}$ & $7,3 \%$ \\
Cita distanciada del tiempo & $2,4 \%$ \\
\hline
\end{tabular}

Fuente: Elaboración propia 
En los casos donde no se consultó al médico, el $39,0 \%$ no hizo nada, el 34,1\% utilizó un remedio casero, $17,1 \%$ se auto recetó, $7,3 \%$ utilizó medico particular y $2,4 \%$ acudió al curandero.

Frente a la necesidad del uso de hospitalización, el 9,6\% de los participantes requirió dicho servicio con ingreso por urgencias, con un tiempo entre la hospitalización y la asignación de cama en el $47,6 \%$ con traslado inmediato, $47,6 \%$ el mismo día y $4,8 \%$ entre 2 a 3 días; el total de los pacientes que acudió a dicho servicio refiere que recibió la atención necesaria en la hospitalización; la atención recibida fue calificada como: muy buena $19 \%$ y buena $81 \%$. Como medio de pago, el 85,7\% utilizó EPS subsidiado y el 14,3\% EPS contributivo.

Finalmente, en cuanto a los gastos de bolsillo, el $8,3 \%$ de los pacientes, debe cancelar cuota moderadora o copago que oscila entre los $\$ 3.000$ hasta los $\$ 40.000$ (media $\$ 8.038$ ), el $24,8 \%$ refiere haber tenido que pagar exámenes, medicamentos o procedimientos que no fueron entregados o autorizados por la EPS que oscila entre los $\$ 3.000$ a $\$ 500.000$ (media $\$ 87.500$ ). Por pago de lentes, audífonos o aparatos ortopédicos 7,5\% lo requirió, con costo entre $\$ 60.000$ a $\$ 350.000$ (media $\$ 177.500)$ y finalmente el pago extra de transporte, alojamiento y alimentación lo requirió el $34,4 \%$ con un costo entre $\$ 2.000$ a $\$ 100.000$ (media \$22.400).

\section{Discusión}

Respondiendo a los objetivos del estudio, se evidenció que la dimensión más relevante dentro de la competencia para el cuidado en el paciente fue la de relación social, de igual manera la dimensión con mejor nivel de competencia en el caso de los cuidadores familiares fue la de anticipación. En términos generales se observó que cerca del $70 \%$ de las diadas son competentes frente al cuidado de la EC. Por otra parte, se identificaron múltiples y variadas barreras de acceso a los servicios de salud en esta población; es de resaltar la barrera de tipo administrativo, ya que implica una gran limitante para las diadas, dadas las características particulares que presenta dicha población. También se convierte en un referente, dado que involucra indirectamente a otras barreras como la económica y la de desplazamiento.

Sin embargo, es relevante acudir a la revisión de otra variables que pueden llevar a una mejor interpretación de este fenómeno de estudio, por ejemplo, el análisis de las variables sociodemo- gráficas de la diada, en donde se destaca que la mayoría de las personas con EC residentes en zona rural, asistentes a los programas de cuidado de paciente crónico en la E.S.Eo de Villa de Leyva, son mujeres, al igual que en el grupo de cuidadores; estas cifras son coherentes a estudios anteriores, en donde las mujeres protagonizan tanto la EC, como el rol de cuidador. Un ejemplo claro, es el estudio realizado por Carrillo et al (16), en donde se evidenció que la mayor proporción de pacientes con EC y cuidadores eran mujeres. Fenómeno que se repitió en el estudio realizado con cuidadores de pacientes con falla cardiaca en la ciudad de Bogotá (17) y de igual manera, en el estudio de Carreño y Arias (18).

También se observó que cerca al $80 \%$ de la muestra se encontraba dentro del grupo etario de adulto mayor ( $\geq 60$ años), lo cual puede ser explicado por el índice de envejecimiento que presenta el municipio. Sin embargo, en estudios previos como el realizado por Aldana et al (19), con pacientes en hemodiálisis, también se evidenció una proporción significativa de paciente adulto mayor. Sin embargo, es de notar que la EC también se hace presente en poblaciones aún más jóvenes, tal como se evidenció en el presente estudio, en correspondencia a la muestra del estudio de medición de competencia para el cuidado a nivel nacional (16).

A diferencia del grupo de pacientes un importante porcentaje de los cuidadores se encontraban entre $\operatorname{los} 35$ a 59 años; valores que se relacionan con los hallazgos del estudio de Carrillo y Carreño (20), realizado en cuidadores de pacientes con cáncer, los cuales se ubicaban en un rango de 18 a 53 años. Es decir que, en su mayoría, los cuidadores familiares se ubican en el grupo etario económicamente productivo, representando un cambio de rol, puesto que, en muchos casos, estos deben dejar de lado sus empleos por asumir el cuidado del paciente. Lo que resulta en una afectación directa en el bienestar económico de la diada y la necesidad sentida por parte del cuidador, de un mayor apoyo de tipo económico por parte de su red de apoyo.

En cuanto al estado civil en el grupo de pacientes, aunque fue más común el matrimonio, se identificó, que sumados aquellos estados en donde la persona no cuenta con un compañero representan una mayor proporción de la muestra, lo cual ha sugerido en otras investigaciones que reduce la capacidad de cuidado en los pacientes. (21) Así mismo, el nivel educativo de las personas con EC en el área rural participantes es bajo, dado por una alta prevalencia de analfabetismo y primaria 
incompleta, reflejando así las desigualdades sociales propias del área rural, las cuales han sido ampliamente estudiadas.

Así, el problema de la educación en el mundo rural tiende a agravar las dificultades relacionadas con la falta de salud, pues si bien es cierto que el porcentaje de analfabetismo general para Colombia es el $8 \%$, este porcentaje es significativamente mayor para las áreas rurales (22), tal como se evidenció en el presente estudio en un $24,3 \%$ de los partcipantes. Asimismo, se debe tener en cuenta que el nivel educativo de la población es un factor determinante relacionado con la salud, la calidad de vida y la autonomía frente a la toma de decisiones en materia de cuidado en salud (23). Un ejemplo claroes el uso de TIC's por parte de la diada, ya que estos no se apoyaban en las TIC's para asumir el cuidado. Esta situación se debe tener en consideración a la hora de proponer intervenciones con uso de TIC's en la población rural. A partir del uso de las TIC's, es posible mejorar la competencia para el cuidado tanto del paciente como de su cuidador, basados en el hecho de que el uso de estas tecnologías, logra garantizar la atención, cobertura y continuidad de los cuidados y la educación en salud en poblaciones remotas o de difícil acceso; además de mejorar los procesos de comunicación entre el paciente y el personal de salud, ofreciendo una alternativa para mejorar su capacidad de gestión frente a la enfermedad y llenando aquellos vacíos de conocimiento frente a la patología.

También se evidenció que en general, el grupo de estudio se ubica en un estrato socioeconómico bajo, con bajos ingresos económicos y afiliación de tipo subsidiado. Este fenómeno se ve explicado por el estancamiento de la generación de empleo en el área rural, llevando a la estabilización de las condiciones de pobreza e inequidad. De ahí que la pobreza sea 2,3 veces mayor en municipios de alta ruralidad comparados a la zona urbana. Esto conlleva a trabajos en condiciones de informalidad que prevalecen en el campo y por ende a que la mayor parte de la población rural se encuentre en calidad de beneficiaria del régimen subsidiado (23).

En conjunto, la diada cuenta con características que aumentan su condición de vulnerabilidad y así mismo la necesidad de intervención, tal como lo plantea el plan nacional de salud rural creado por el Ministerio de Salud colombiano, en donde se pretende priorizar las intervenciones dirigidas hacia el envejecimiento para así mejorar la protección y atención de los adultos mayores (24).

En cuanto a la capacidad de cuidado en la rura- lidad, medida a través de la competencia para el cuidado en las personas con EC, se evidencio que a pesar de las fuertes dificultades en todos los ámbitos a las que esta población se ve enfrentada, cerca del $60 \%$ de la muestra obtuvo un nivel óptimo en cuanto a su competencia para el cuidado. Sin embargo, considerando que son una población vulnerable que además padece una patología crónica, este porcentaje no es suficiente. Haciendo necesaria la intervención continua del personal de salud, tanto en el seguimiento de la adherencia a los programas de salud dirigidos a este grupo poblacional como en la planeación del desplazamiento del personal a las áreas de difícil acceso para garantizar la atención necesaria.

Dentro de los puntos favorables encontrados en la medición, se observó el adecuado nivel en la dimensión disfrutar, la cual hacen referencia a la percepción de bienestar y calidad de vida a pesar de la enfermedad. Aun cuando la EC es bastante limitante, las personas con EC que hicieron parte de la muestra reconocen que su calidad de vida es buena. Esto, se encuentra estrechamente relacionado al hecho de que los pacientes son más competentes en la dimensión relación social e interacción, dada su capacidad de relacionarse de manera efectiva con sus redes de apoyo y de manera especial con su cuidador familiar primario, lo cual contribuye a un mejor desempeño en la labor de cuidado. Dichas dimensiones pueden verse favorecidas, si desde el rol de enfermería, se involucra a los cuidadores en encuentros sociales que permitan el fortalecimiento de las redes de apoyo, así como la identificación del agotamiento de cuidadores que son incompetentes en estas áreas.

Adicionalmente, gracias a las óptimas redes de apoyo con las que cuenta el paciente y adecuada relación de cuidado con su cuidador (es), han desarrollado satisfacción personal a partir de su cuidado y su condición de salud. Así, las personas con EC entrevistadas relacionaron la aceptación de su patología a su proceso vital y refirieron que el apoyo espiritual que reciben es de gran ayuda a la hora de afrontar su EC. Considerando que la población boyacense cuenta con una fuerte espiritualidad, esta relación deberá ampliarse en estudios futuros.

Frente a todo lo anterior, cabe resaltar que a pesar de la favorable proporción de pacientes con EC con alto nivel en la competencia para su cuidado en el hogar; se encontraron puntos álgidos como las dimensiones unicidad y conocimiento. Esto se traduce en que los pacientes crónicos participantes, cuentan con insuficientes conocimientos 
básicos sobre su condición de salud, así como sobre el manejo médico instaurado que deben seguir para reducir el riesgo de recaídas y complicaciones, lo que conlleva a falta de adherencia a los manejos médicos.

Dicho fenómeno, podría ser explicado a partir del bajo nivel educativo que presenta este grupo. Un $24,3 \%$ de ellos era analfabeta, lo cual nos lleva a cuestionar la efectividad de las intervenciones dirigidas a esta población. Educar a este grupo poblacional es especialmente difícil si no se caracteriza en primera instancia. De igual manera, se reconocen las limitaciones ya mencionadas en la ruralidad, como el escaso número de personal sanitario, lo cual reduce el tiempo de intervención en la consulta, que podría ser dedicado a la educación del paciente para el conocimiento y reconocimiento de su enfermedad. Frente a lo cual, se hace necesaria la inmersión del personal de enfermería en la realidad de la población, a la vez que se debe hacer participe al paciente en la estructuración de los programas educativos generados por la institución de salud.

De esta manera, reconocemos como los retos del cuidado en la ruralidad de la persona con EC, se basan en la necesidad sentida de educación sobre su patología, tratamiento y posibles complicaciones, esto con el fin de reducir el desenlace catastrófico que puedan tener las patologías crónicas sobre esta población y, por otra parte, se reconoce como el nivel educativo repercuten la capacidad del sujeto para ejercer su cuidado.

En cuanto al cuidador familiar, se identificó que es más competente para el cuidado en comparación con el paciente. Este hallazgo es comparable con las investigaciones que incluyen a la diada, tales como la realizada por Carrillo et al (16), con diadas de cuidador familiar y persona con EC a nivel nacional y de igual manera, se evidencio esta diferencia entre los actores de la diada, en el estudio realizado con personas con cáncer en quimioterapia y sus cuidadores (6).

Respecto a los aspectos positivos en la competencia para el cuidado, se encontraron las dimensiones anticipación y disfrutar. Estas dimensiones hacen referencia a la capacidad de resiliencia que presenta el cuidador. En este caso es buena, puesto que la calidad de vida y la satisfacción personal con la que este cuenta, medidas desde la perspectiva de la competencia para el cuidado son buenas, sin embargo, se debe tener presente que dichos tópicos pueden llegar a verse afectados luego de un desarrollo prolongado del rol como cuidador. Estas dimensiones también se encuentran bien representadas en estudios rea- lizados con cuidadores de paciente con cáncer (20).

Las dimensiones con niveles más bajos fueron conocimiento, instrumental y relación social, es decir que los cuidadores familiares del estudio presentan deficiencias frente a los conceptos básicos de la enfermedad y de igual manera, reflejan bajos conocimientos en las habilidades propias del cuidado. Esto podría relacionarse con el bajo porcentaje de cuidadores familiares que están a cargo de la persona crónica desde su diagnóstico, puesto que el tiempo como cuidadores mejora los conocimientos y habilidades frente al cuidado (25). Por ultimo y en contraposición a los hallazgos encontrados en la competencia de la persona con EC, la dimensión relación social es una de las más bajas en el grupo de cuidadores, lo cual podría relacionarse con lo referido por ellos mismos, dado el limitado apoyo que perciben por parte de otros familiares a la hora de asumir el cuidado de la persona con EC.

Por último, se reconoce que aún se deben fortalecer algunas dimensiones en la diada, reconociendo la particularidad de cada uno de sus participantes, puesto que en el grupo de personas con EC que hicieron parte de la investigación, se debe reforzar lo concerniente a las dimensiones conocimiento y unicidad, y por parte del grupo de cuidadores familiares se debe fomentar las dimensiones conocimiento, instrumental y relación social.

Ante este panorama, se hace necesaria e indispensable la intervención del profesional de enfermería, ya que la educación del paciente es un aspecto fundamental de la atención de salud y cada vez es más reconocido como una función esencial en la práctica de enfermería (26), lo cual es más que necesario en una comunidad en condición de vulnerabilidad como lo es la zona rural.

De esta manera, la enfermera (o) rural desarrolla un rol trascendental en la gestión de los servicios y programas de salud, dado que aborda el proceso de salud desde el análisis y priorización de la situación hasta la evaluación y control de las acciones sanitaria (27), dicho proceso debe fundamentarse en resultados de caracterización de la población rural, con el fin de ofrecer una atención más efectiva y de calidad. La enfermera (o), debe aprovechar su rol y reconocimiento dentro de la comunidad, como se ha revelado en algunos estudios, ya que es evidente que las personas valoran el consejo y la opinión de la enfermera (o) en el cuidado o recuperación de la salud y se percibe como una instancia de confianza y apoyo (27). 
De esta manera, el trabajo del profesional en el aumento de la concienciación sobre la salud de las poblaciones con enfermedades crónicas y de alto riesgo, no sólo ayudaa las personas a conocer más sobre las enfermedades y aumentar su capacidad para el autocuidado, sino que también les ayuda a dejar conductas no saludables y llevar una vida saludable (28).

La evaluación del acceso que presenta la diada a los servicios de salud fue realizada de acuerdo con diferentes niveles: en primer lugar, el acceso a los servicios preventivos, los cuales en su mayoría son ofrecidos por la E.S.E. de primer nivel donde asisten mensualmente a sus controles de patología crónica, en los cuales se observa un gran porcentaje de adherencia ya que estos servicios se prestan alrededor de la consulta de control crónico a la que acuden mensualmente. Al comparar los hallazgos con el estudio realizado por Arrivillaga (29) en el municipio de Jamundí, Valle, se evidenció que aquellos servicios correspondientes a prevención, realizados en las instituciones de salud, cuentan con alto porcentaje de adherencia.

En contraste, frente a aquellos procedimientos que no ofrece la institución de atención primaria, se mostró muy baja adherencia, lo cual es justificado por la necesidad de desplazamiento de los pacientes a la ciudad capital del departamento para la toma de estos, razón por la cual refieren no acceder a ellos.

Por otra parte, y como se ha evidenciado ampliamente en la revisión bibliográfica, el distanciamiento de los centros de salud en la población rural influye de manera importante en el acceso a los servicios, de ahí que se identificaran casos en donde la diada contaba con un tiempo de desplazamiento de incluso casi 4 horas. En cuanto al medio de desplazamiento utilizado, gran proporción emplea la caminata, la cual más allá de un factor protector para la EC, se convierte en el único recurso con el que cuenta la persona con EC y su cuidador familiar, para lograr acceder a la institución; condición que se hace más compleja de realizar cuando el paciente con EC es un adulto mayor con limitaciones para la movilidad.

Adicional a lo anterior y teniendo en cuenta que la diada cuenta con bajos recursos económicos, el considerar el uso de vehículo de transporte público, adiciona un gasto de bolsillo importante a la diada, sumado a lo cual, ellos refieren que el sistema de transporte también es precario en el municipio, dado por el estado de las vías, el reducido número de unidades móviles y la demora en los traslados.
En cuanto al acceso a los servicios curativos, los cuales incluyen la consulta médica ofrecida por la E.S.E. de primer nivel y la atención médica especializada que se ofrece fuera del municipio, se encontró una alta adherencia a las citas por medicina general, dada la alta oferta de la institución; en contraposición, en los casos en los que los pacientes no acudieron a consulta por medicina general, se encontró que los aspectos que influyeron fueron principalmente tramites excesivos y dispendiosos, mala atención del personal administrativo, el centro de atención queda lejos o falta de dinero para transportes y copagos. Los cual refleja de nuevo las limitantes de tipo administrativo y económico.

Ahora bien, la situación frente a la atención con medicina especializada no es completamente diferente, ya que el tiempo de espera entre la solicitud de la cita por el médico general y la autorización de la misma es variado, encontrándose entre 1 a 4 semanas, llegando incluso a superar el mes; sumado lo anterior, este no es el único trámite al que se ve enfrentada esta población, puesto que luego de la autorización, existe otro periodo de espera entre la autorización y la consecución de la cita, que en la mayoría de los casos va desde 2 semanas hasta 3 meses, tiempo en el cual según la diada, se vencen las autorizaciones dadas por la EPS y/o paraclínicos de control que deben ser entregados para evaluación por parte del especialista, llevando al retroceso en el proceso y por ende la necesidad de iniciar nuevamente el trámite, generando retrasos en el proceso de atención del paciente e incluso una atención inadecuada para la condición de salud actual del mismo, puesto que el lapso de tiempo es muy prolongado.

Cabe mencionar que la atención del especialista solo es prestada en la ciudad capital del departamento, lo cual adiciona el desplazamiento del municipio a la cuidad capital para recibir la atención por medicina especializada, representando un gasto de bolsillo adicional.

Así mismo, en la investigación de Arrivillaga et al (29), se reportaron demoras respecto al proceso de atención por parte de médico especialista, en especial en el tiempo de autorización por parte de la EPS, en donde el 48,6\% le llevó de 1 día a 1 semana; al 36,8\% de la muestra de 1 a 4 semanas y en el 14,7\% de los casos más de 1 mes. Comportamiento que se repite al momento de medir el tiempo de cita efectiva con el médico especialista, en el cual el tiempo de espera entre la autorización y la cita fue de 1 día a 1 semana en el $23,7 \%$ de los participantes, de 1 a 4 semanas 
en el 52,7\% y de 1 a 3 meses en el 21,8\%. Patrón acorde a lo hallado en la presente investigación, mostrando así las barreras administrativas causadas por la atención de la EPS.

Este es un claro reflejo de una de las características del mundo rural latinoamericano en la que los servicios de salud están sobre todo en las áreas urbanas y tienen muy poca presencia en las pequeñas poblaciones y en las regiones con población dispersa (23). Así, los retos a los que se ven enfrentada esta población a menudo hacen referencia al acceso a la atención especializada y a las autorizaciones que retrasan la atención, adicional a esto, en otras investigaciones, los usuarios de la zona rural añaden costes en tiempo y transporte, para hacer el trámite y la obtención de autorizaciones (30); generando así barreras de acceso a la población a los servicios de salud, tales como barreras administrativas y de desplazamiento.

De igual manera, en cuanto al gasto de bolsillo, el cual evalúa principalmente las barreras económicas que se presentan en la atención, se encontró la necesidad de aporte económico en diferentes aspectos como compra de medicamentos, esto derivado de los casos donde la EPS no entregaba la totalidad de la medicación prescrita, como consecuencia a la exclusión de un determinado medicamento en el POS o por no contar con el medicamento en la E.S.E. de primer nivel donde es atendido el paciente. Todo esto conlleva a interrupciones en el tratamiento médico e incluso posible abandono de este. Complementando lo anterior, la diada informó sobre requerimiento de copagos los cuales se presentan en aquellos pacientes afiliados al régimen contributivo.

Finalmente, los gastos en transporte y/o alimentación son los más prevalentes en la diada, ya que se encuentran relacionados con el requerimiento de desplazamientos tanto a la institución de primer nivel que presta la atención en el municipio, como a las oficinas de las EPS para la realización de los trámites administrativos y también al traslado a la ciudad capital del departamento, para recibir la atención médica especializada o recepción de medicamentos que no son entregados en el centro de atención de referencia. Al asociar estos gastos con las condiciones económicas de la diada, se reconoce que la obligación de estos pagos se transforma en una barrera importante de acceso a los servicios de salud.

Desde este punto de vista y dado que la ruralidad constituye un importante factor de riesgo de acceso a los servicios, brindar cuidados integrales y continuos desde una perspectiva cultural, considerando que en el ámbito rural existen múlti- ples formas de cuidado cultural tradicional, es una tarea primordial de la enfermera(o) Además de reconocerse como la poseedora de la capacidad de resolución de problemas in situ en forma oportuna y eficiente con el objetivo de gestionar el acceso de la población a la red asistencial (27). Brindar información de estos fenómenos desde la perspectiva de los pacientes y sus cuidadores, ofrece una visión amplia al profesional de enfermería, de la necesidad sentida de una intervención mucho más allá de la obligación institucional, puesto que implica hacer uso del conocimiento que se tiene sobre las condiciones especiales de la población a intervenir; además de conocer las limitantes propias de la misma. Permite reinventar la atención que ofrece la enfermera (o), con el fin de subsanar en alguna medida, las deficiencias identificables a partir de la información que aportan los mismos usuarios del sistema sobre las problemáticas en salud, incluso son ellos mismos quienes pueden llegar a ofrecer soluciones por medio del trabajo en equipo con el mismo personal que les atiende.

Sin embargo, no se pueden negar las evidentes limitaciones con las que cuenta la enfermera (o), que van más allá que de su quehacer, puesto que para minimizar o eliminar por completo las barreras de acceso a los servicios de salud, se requiere un trabajo intersectorial, que incluya los ejes centrales que dirigen y manejan el sistema de salud mismo.

Por ende, la enfermera (o) se convierte en muchas ocasiones, en el enlace entre las comunidades y las instituciones de salud, y a la vez tiene la posibilidad de visibilizar las condiciones de acceso a las cuales se ven enfrentados los pacientes. De esta manera, su reconocimiento sobre las necesidades de otros se constituye, en una herramienta práctica a la hora de liderar las modificaciones necesarias en los ámbitos rurales, frente a la atención en salud de las comunidades. Así, la enfermera (o) salvaguarda de una u otra manera el derecho a la salud y está obligada(o) a promover iniciativas de cambio frente al mismo equipo de salud y a diferentes instancias con el fin de lograr un impacto positivo en la atención y cuidado de los enfermos crónicos rurales.

En conclusión, la identificación de las barreras de acceso a los servicios de salud en la ruralidad es fundamental ya que permite a los hacedores de políticas públicas y directores de programas municipales, detectar en dónde se encuentran las principales limitantes de acceso; para asi establecer los correctivos pertinentes para garantizar el uso de servicios de salud de manera oportuna, fa- 
cilitando el cuidado en la salud de la población. Además, permite aportar conocimiento desde la perspectiva de los usuarios del sistema, sobre los aspectos que determinan el acceso, ya que son escasos los estudios que ahondan en este tópico (31).

\section{Conclusiones}

- El principal reto por asumir frente al cuidado de la enfermedad en la zona rural, por parte del paciente con EC y su cuidador, son los limitados conocimientos con los que cuentan para encarar la enfermedad crónica y las condiciones de vida que dificultan la obtención de estos.

- A pesar de las diferentes limitaciones generadas en la ruralidad, el profesional de enfermería, cuenta con una herramienta muy útil para generar el cambio frente los retos revelados en las deficiencias de la competencia para el cuidado de los pacientes con EC y sus cuidadores, la educación; pues es reconocido su liderazgo por distintos actores en el ámbito de la educación a las comunidades, y a la vez cuenta con la facilidad del contacto constante, tanto con los individuos como con las comunidades.

- Los retos que asume la diada rural en cuanto al acceso a los servicios de salud derivan de las barreras de acceso a los mismos, dentro de las cuales están las identificadas en el estudio que fueron las barreras administrativas, económicas y de desplazamiento.

- La enfermera (o) en zona rural, es indispensable a la hora de identificar los retos existentes en el proceso de cuidado de la enfermedad crónica y a la vez en el acceso a los servicios de las personas que padecen estas patologías, reconociendo al profesional como el ente idóneo para generar procesos de cambio.

- Dado el tipo de atención que se ofrece a las diadas, es de gran utilidad hacer uso de los programas de cuidado a persona con EC, con el fin de fortalecer en especial los deficientes conocimientos con los que cuenta la diada, lo cual mejorará en importante medida su competencia para cuidar. De igual manera, en caso de considerar el uso de TIC's, deben ser tenidos en cuenta los enfoques pedagógicos integrales para garantizar un cuidado más acorde a los contextos sociales y culturales.

\section{Conflicto de Intereses}

Los autores declaran no tener ningun conflicto

de intereses.

\section{Referencias bibliográficas}

1. Goeres LM, Gille A, Furuno JP, Erten-Lyons D, Hartung DM, Calvert JF, et al. Rural-Urban Differences in Chronic Disease and Drug Utilization in Older Oregonians. J Rural Health [Internet]. 2016 [Consultado 30 enero 2020];32(3):269-79. Available from: https://doi.org/10.1111/ jrh. 12153

2. Lauckner HM, Hutchinson SL. Peer support for people with chronic conditions in rural areas: a scoping review. Rural Remote Health [Internet]. 2016 [Consultado 25 enero 2020];16(1):3601. Available from: https://pubmed.ncbi.nlm.nih.gov/26943760/

3. Paskulin LMG, Molzahn A. Quality of Life of Older Adults in Canada and Brazil. West J Nurs Res [Internet]. 2007 [Consultado 22 marzo 2020]; 29(1):10-26. Available from: https://doi. org/10.1177\%2F0193945906292550

4. Hayter AKM, Jeffery R, Sharma C, Prost A, Kinra S. Community perceptions of health and chronic disease in South Indian rural transitional communities: a qualitative study. Glob Health Action [Internet]. 2015 [Consultado 22 marzo 2020]; 8(1):25946. Available from: https://doi. org/10.3402/gha.v8.25946

5. Carrillo-González GM, Barreto RV, Arboleda LB, Gutierrez-Lesmes OA, Gregoria-Melo B, Tamara-Ortiz V. Competencia para cuidar en el hogar de personas con enfermedad crónica y sus cuidadores en Colombia. Rev la Fac Med. 2015;63(4):669-75. 
6. Sánchez LM, Mabel Carrillo G. Competencia para el cuidado en el hogar diada persona con cáncer en quimioterapia-cuidador familiar. Psicooncologia. 2017;14(1):137-48.

7. Quintero D. Patricia S. Sobre una propuesta de popularización del derecho a la salud con comunidades rurales 1 . A proposal about the popularization to the right of health with rural communities. 2016;12.

8. Bejarano-Daza JE, Hernández-Losada DF. Fallas del mercado de salud colombiano. Rev la Fac Med [Internet]. 2017 [Consultado 22 noviembre 2019]; 65(1):107-13. Available from: https:// doi.org/10.15446/revfacmed.v65n1.57454

9. Huang X, Yang H, Wang H, Qiu Y, Lai X, Zhou Z, et al. The Association Between Physical Activity, Mental Status, and Social and Family Support with Five Major Non-Communicable Chronic Diseases Among Elderly People: A Cross-Sectional Study of a Rural Population in Southern China. Int J Environ Res Public Health [Internet]. 2015 [Consultado 22 noviembre 2019];12(12):13209-23. Available from: https://doi.org/10.3390/ijerph121013209

10. Casado-Cañete F. Objetivos de desarrollo del milenio. Informe Universidad Politecninca de Catalunya, Epaña [Internet]. 2015 [Consultado 22 noviembre 2019]; 56-57. Available from: https:// upcommons.upc.edu/handle/2099/7274

11. Informe nacional de calidad de la atención en salud 2015. Ministerio de salud y protección social Colombia. 2015. Available from: https://www.minsalud.gov.co/sites/rid/Lists/BibliotecaDigital/ RIDE/DE/DIJ/informe-nal-calidad-atencion-salud-2015.pdf

12. Boletín Técnico de Pobreza Multidimensional en Colombia Año 2018. DANE. Available from: https://www.dane.gov.co/files/investigaciones/condiciones_vida/pobreza/2018/bt_pobreza_ multidimensional_18.pdf

13. Restrepo-Zea JH, Silva-Maya C, Andrade-Rivas F, Vh-Dover R. Acceso a servicios de salud: Analisis de barreras y estrategias en el caso de Medellin, Colombia. Rev Gerenc y Polit Salud. 2014;13(27):236-59.

14. Maimela E, Alberts M, Modjadji SEP, Choma SSR, Dikotope SA, Ntuli TS, et al. The Prevalence and Determinants of Chronic Non-Communicable Disease Risk Factors amongst Adults in the Dikgale Health Demographic and Surveillance System (HDSS) Site, Limpopo Province of South Africa. PLoS One [Internet]. 2016 [Consultado 22 noviembre 2019];11(2):e0147926. Available from: https://doi.org/10.1371/journal.pone.0147926

15. Organización Panamericana de la Salud. Ampliación del rol de las enfermeras y enfermeros en la atención primaria de salud. Washington, D.C.: OPS; 2018.

16. Carrillo-González GM, Barreto-Osorio RV, Arboleda LB. Competencia para cuidar en el hogar de personas con enfermedad crónica y sus cuidadores en Colombia. Bogota, Rev. Fac. Med. 2015 Vol. 63 No. 4: 665-75

17. Achury-Saldaña DM, Restrepo-Sánchez A, Torres- Castro NM, Buitrago-Mora AL, Neira-Beltrán NX , Devia-Florez PD. Competencia de los cuidadores familiares para cuidar a los pacientes con falla cardíaca. Rev Cuid. 2017; 8(3): 1721-32.

18. Carreño-Moreno S, Arias-Rojas M. Competencia para cuidar en el hogar y sobrecarga en el cuidador del niño con cáncer. Gac Mex Oncol. 2016; 15(6):336-43.

19. Aldana EA, Barrera SY, Rodríguez KA, Gómez OJ, Carrillo GM. Competencia para el cuidado (CUIDAR) en el hogar de personas con enfermedad renal crónica en hemodiálisis. Enfermería Nefrológica [Internet]. 2016 [Consultado 22 noviembre 2019];19(3):3-9. Available from: http:// scielo.isciii.es/scielo.php?script=sci_arttext\&pid=S2254-28842016000300009\&lang=pt

20. Carrillo GM, Carreño SP, Sánchez LM. Competencia para el cuidado en el hogar y carga en cuidadores familiares de adultos y niños con cáncer. Revista Investigaciones Andina. 2018; 20(36):87-101.

21. Girardi-Paskulin LM, Molzahn A. Quality of Life of Older Adults in Canada and Brazil. West J Nurs Res [Internet]. 2007 [Consultado 22 marzo 2020]; 29(1):10-26. Available from: https:// 


\section{doi.org/10.1177\%2F0193945906292550}

ISSN-PRINT

$1794-9831$

E-ISSN 2322-7028

Vol. 17 No. 3

Sep - Dic 2020

Cúcuta, Colombia
22. Pérez-Correa E. Nueva ruralidad, globalizacion y salud. Rev CES Med 2007; 21(Supl 1):89-100

23. Pineda BC. Desarrollo humano y desigualdades en salud en la población rural en Colombia. Univ Odontol. 2012 Ene-Jun; 31(66): 97-102.

24. Republica de Colombia. Plan Nacional de Salud Rural Ministerio de Salud y Protección Social [Internet] 2018; Available from: https://www.minsalud.gov.co/sites/rid/Lists/BibliotecaDigital/ RIDE/DE/PES/msps-plan-nacional-salud-rural-2018.pdf

25. Coppetti LC, Girardon-Perlini NMO, Andolhe R, Gutiérrez MGR, Dapper SN, Siqueira FD. Caring ability of family caregivers of patients on cancer treatment: associated factors. Rev. Latino-Am. Enfermagem. 2018; 26:e3048.

26. Soto P, Masalan P, Barrios S. La educación en salud, un elemento central del cuidado de enfermería. ELSEVIER. 2018; 29 (3):288-300.

27. Marilaf M, Alarcón A, Illesca M. Rol del enfermero/a rural en la región de la Araucanía Chile: percepción de usuarios y enfermeros. Ciencia y Enfermería. 2011 (2):111-118.

28. Tian M, Wang H, Tong X, Zhu K, Zhang X, Chen X. Essential public health services' accessibility and its determinants among adults with chronic diseases in China. PLoS One. 2015;10(4):1-12.

29. Arrivillaga M, Aristizabal JC, Pérez M, Estrada VE. Encuesta de acceso a servicios de salud para hogares colombianos. Gac Sanit [Internet]. 2016; 30(6):415-20. Available from: http://dx.doi. org/10.1016/j.gaceta.2016.05.008

30. Campaz N, Montaño SM. Barreras de acceso al servicio de salud en el contexto colombiano a partir de la promulgación del derecho a la salud en la legislación colombiana. Universidad Santiago de Cali; 2019.

31. Acosta SR. Barreras y Determinantes del Acceso a los Servicios de Salud en Colombia. [Tesis Especialización]. Popayán: Universidad EAN - Universidad del Cauca; 2010. 